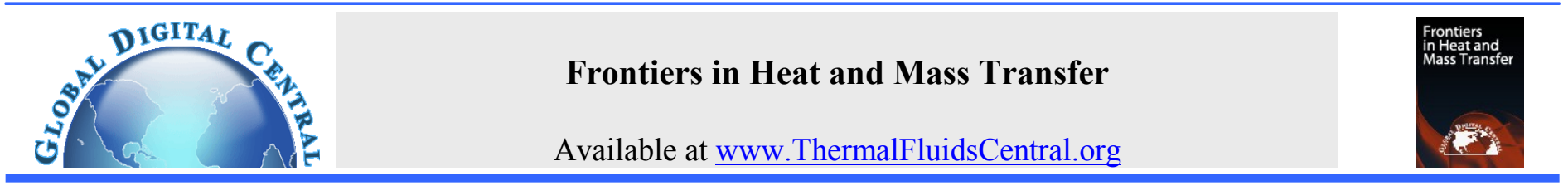

\title{
EX VIVO LIVER TISSUE RADIOFREQUENCY THERMAL ABLATION: IR MEASUREMENTS AND SIMULATIONS
}

\author{
Edoardo Gino Macchi*, Giovanni Braschi, Mario Gallati \\ Università di Pavia, Dipartimento di Ingegneria Civile ed Architettura, via Ferrata 3, 27100 Pavia (PV), Italy
}

\begin{abstract}
Radiofrequency thermal ablation (RFTA) is a medical procedure currently widely adopted for liver tumors treatment. Its outcome is strongly influenced by temperature distribution near the RF applicator therefore continuous measurements are required both to validate RFTA numerical models and to better control the outcome of the procedure. The space-time evolution of the thermal field during RFTA on ex vivo porcine liver tissue has been measured by infrared thermal imaging in different experimental setups. A three-dimensional simulation of the whole experiment reproduces all the features of the thermal field measurements and validates the proposed measurement methodology.

Keywords: radiofrequency ablation, ex vivo liver tissue, IR temperature measurements, mathematical model, numerical simulations
\end{abstract}

\section{INTRODUCTION}

In the last 10 years, many works in medical literature have highlighted the importance of radiofrequency thermal ablation (RFTA) in the treatment of liver tumors. The practice, pioneered by Rossi et al. (1990) and McGahan et al. (1992), consists in necrotizing the tumor tissue by means of a localized Joule heating produced by a RF electrical field applied between a small active electrode (needle), placed in the centroid of the tumor, and an external electrode with great surface area positioned on the skin. Above about $60^{\circ} \mathrm{C}$ proteins denaturation (and therefore cell death) takes place almost instantaneously (Goldberg et al., 1995; van Sonnenberg et al., 2005); when boiling temperature is reached the liquids start evaporating. Vapor bubble generation close to the active electrode breaks the electrical continuity between the needle and the tissue, and stops the procedure.

The literature has highlighted both the merits of the technique (e.g., ease of use, minimally invasive) and its limits (e.g., procedure duration limited by vaporization). The dimension of treatable tumors has progressively grown using cooling and extensible tips of various shapes and today lesions of $3-3.5 \mathrm{~cm}$ can be often achieved (van Sonnenberg et al., 2005). Still several challenges must be faced. The main challenges deal with: (1) how to get larger lesions and control their development and outcome, (2) how to apply the method to tissues physically unfit for RFTA (poorly electrically conductive tissues), (3) how to input power efficiently and with even less invasive methods.

The numerical simulation of the phenomenon can help in such a context: rather than a tool to foresee the outcome of a particular treatment, the numerical simulation should be seen as a method useful to study the effects of particular biological situations and to preliminary test new ways to improve the RFTA procedure. RFTA modeling is a complex task since, in order to correctly represent the phenomena, liquid-vapor phase change must be modeled. Both the RF ablation procedure duration and the temperature distribution inside the tissue are fundamental data needed to validate any RFTA model.
This work is focused on the temperature distribution measurement during RFTA using thermal imaging. IR measurements are used both to qualitatively outline the heating dynamics around the active tip and to further validate a previously calibrated model. Numerical modeling is then employed to check both the physical consistency of the measurements and to validate the measurement methodology.

\section{EXPERIMENTAL TESTING}

\subsection{Thermal field measurements strategies}

The reference experiments must preserve the main features of the medical procedure such as space and time scales and the operational constraints: the RF generator, the power applied and the dimensions of the needle are similar to those employed in medical practice. The electromagnetic field frequency is $480 \mathrm{kHz}$, while the power switch off is automatic, when the electrical impedance $Z$ exceeds $300 \Omega$. The porcine liver specimens came from a local slaughterhouse.

The ideal reference geometry to carry RFTA tests is usually 3D and axisymmetric with the active electrode placed at the center of mass of the cylindrical testing volume of liver tissue. Theoretically the use of traditional temperature sensors allows one to get the temperature time evolution at any specific point, however, from the practical point of view, it is nearly impossible to get a meaningful evaluation of the strong temperature gradients (up to $7^{\circ} \mathrm{C} / \mathrm{mm}$ ) with measurements in close points, due to the sensor size and placement uncertainty. Furthermore the intense EM field near the active electrode often produces a strong EM noise that may even make the measurements meaningless. For this reason in most experiments found in the literature the tissue temperature is measured only in a few points, usually far from the active electrode, to avoid both strong EM interferences and alterations to the RF field that produces the tissue heating (Yang et al., 2007; Ai et al., 2012; Trujillo et al., 2012; Gallati and Braschi, 2013). While standard fiber optic sensors (fiber Bragg gratings) solve the 
problems related to size and EM interferences, they provide a temperature measurement averaged over their sensing length (usually 0.5-1 cm) (Tosi et al., 2014), and therefore are not very suited for capturing the steep temperature gradients induced by most thermal ablation treatments. On the other hand, infrared (IR) imaging has the advantage to provide high resolution field measurements avoiding the thermal and electrical effects due to the sensors presence that affect the system under measurement and it is insensitive to electromagnetic noise. However the need for a clear line of sight to the emitting surface often fights against the energy balance preservation.

In the present investigation the $3 \mathrm{D}$ sample is sectioned along the axial plane; the sample surface can be left uncovered, to limit any energy filtering effect along the line of sight, or it can be protected with a thermally insulating lid. In case the "free surface" solution is being adopted significant thermal losses are expected, as heat and vapor can freely leave the sample, and, moreover, the surface emissivity might vary considerably as the water content dramatically changes during the procedure. On the other hand, with the insulating lid, thermal losses are substantially reduced and the surface emissivity is unchanged, however it is hard to find a material exhibiting at the same time the proper thermo-mechanical properties and the needed infrared total transparency. A compromise solution has been adopted: the temperature is measured only at some points, relying on an array of highly conductive thin copper nails (diameter $0.6 \mathrm{~mm}$ ) piercing through an insulating balsa wood lid. It is expected that these nails produce a heat bypass from the tissue towards the outside only in a few points, therefore limiting any loss and preserving the overall thermal balance; the temperature of the nails is then measured by IR imaging.

The axial setup with free surface, allows one to simultaneously record the phenomena evolution during the RFTA in both visible and IR range: as stated above, due to the significant heat losses, the results can be used only from a qualitative point of view. On the other hand if the lid with nails is employed the losses are greatly reduced and the temperature measurements could be used from a quantitative point of view.

\subsection{Experimental setup and measurements}

The test box is shown in Fig. 1: it is a hollow half cylinder, having diameter $D$ and length $s l$, carved out of a single block of insulating material (extruded polystyrene, XPS). Porcine liver tissue $(L)$ fills the box cavity. The cavity wall is covered with a thin brass coating, acting as the passive electrode ( $p e$ ). For the free surface experiment the active electrode (ae) is the tip of a thin brass pipe, having $4 \mathrm{~mm}$ outer diameter and $20 \mathrm{~mm}$ active length, while the rest of the pipe is electrically insulated (Fig. 1a).

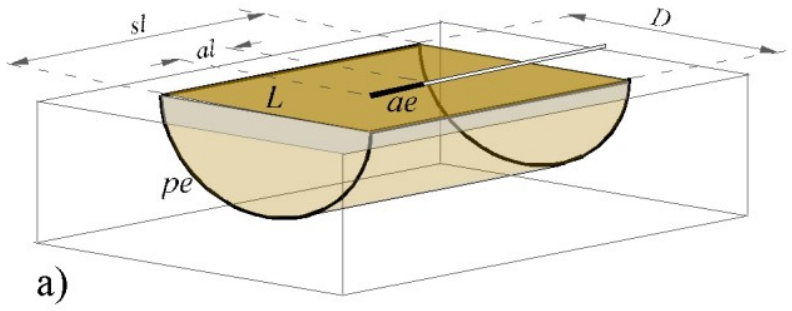

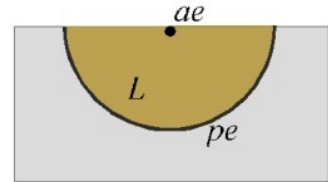

b)

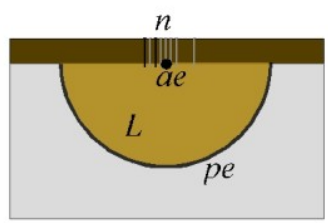

c)
Fig. 1 RFTA test box: a) axonometric view, free-surface setup; b) vertical cross section, free-surface setup; c) vertical cross section, setup with nail-equipped lid. ae: active electrode, pe: passive electrode, $t$ : thermistor, $L$ : liver, $a l=20 \mathrm{~mm}, D=80 \mathrm{~mm}$, $s l=\sim 100 \mathrm{~mm}$.
In the tests with nail-equipped lid the active electrode is directly fixed to the balsa wood lid (see Fig. 1c and Fig. 2); the cover also carries a NTC thermistor. A schematic of needle, nails numbering and NTC position is depicted in Fig. 2 together with a picture of the insulating lid near the active electrode (inner side). The outer surface of the lid (both nails and balsa wood) was painted black to get higher and more homogeneous surface emissivity. The emissivity was estimated using a small copper cube of $3 \mathrm{~cm}$ side, previously black-painted, and equipped with several micro-thermocouples (both on the surfaces and inside the cube). After heating the cube using a heat gun, temperature measurements were performed using both thermocouples (that give the actual temperature) and IR imaging. The surface emissivity was corrected until IR temperature measurements and thermocouple measurements (those recorded on the surface) matched. Using this method, black-painted copper emissivity, was estimated to be 0.86 , with a computed uncertainty of about $1.5 \%$.

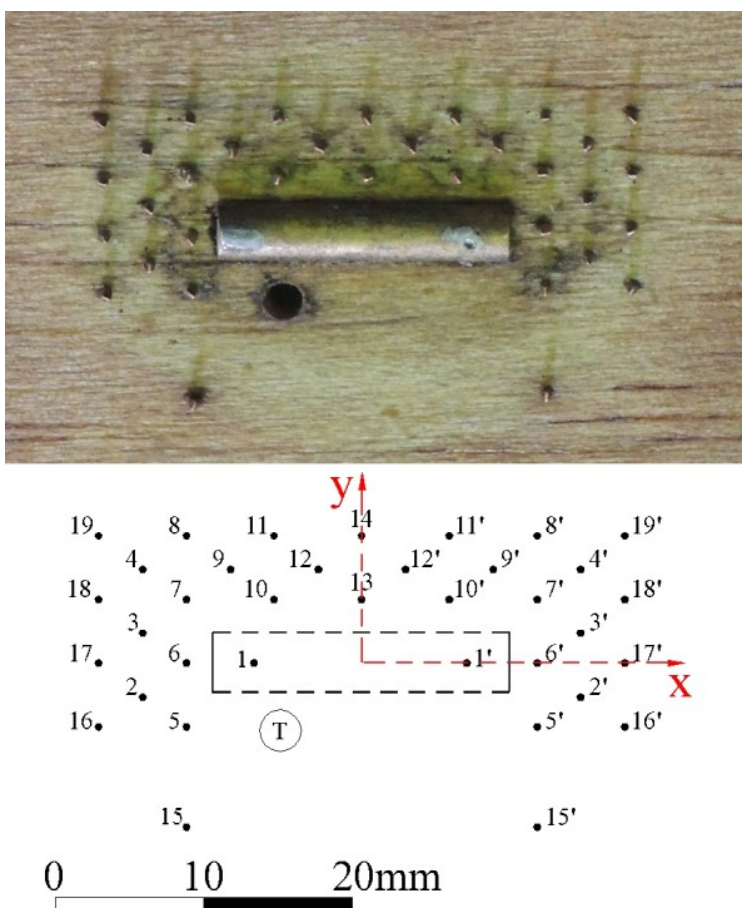

Fig. 2 Balsa wood lid with copper nails, detail near the active tip: picture (top) and schematic drawing (bottom) of nails pattern, active electrode and thermistor position (T).

With the above described box, laid down horizontally, videos were recorded from the top, by means of an IR camera. The main characteristics of the employed IR camera (Thermo Tracer TH7100 NEC, Tokyo, Japan) are: resolution $320 \times 240$ pixel, spectral band 7.5 to $13 \mu \mathrm{m}$, temperature range -20 to $+120^{\circ} \mathrm{C}$, uncertainty $\pm 2{ }^{\circ} \mathrm{C}$ or $\pm 2 \%$ of reading, frame rate $10 \mathrm{~Hz}$. The IR images were processed by means of custom developed Matlab ${ }^{\circledR}$ scripts. A temperature value was extracted from each pixel, the nails temperature was computed as the average of the pixels belonging to the area occupied by each nail. Since traditional temperature sensors (thermocouples, RTDs, etc.) can be more sensitive to EM noise than NTC thermistors, the latter (B57861 Epcos, Munich, Germany) were employed to check the IR measurements.

The temperature measurements obtained using the IR camera may be subject to many uncertainty sources which are part of the measurement process and not just related to the IR camera uncertainty. Being conscious of the potentially large bias between liver and measured temperatures a proper uncertainty analysis was carried out (JCGM 100, 2008). The approach followed consists in evaluating all the uncertainty contributions, laying down an uncertainty budget, and defining a combined uncertainty of the measurements system (IR 
camera plus nail-equipped balsa wood lid). The main uncertainty sources are related to: IR camera, emissivity of the surface, dynamic response of the measurement system (nails included) and temperature gradient along the nail. The resulting expanded uncertainty is about $5.5^{\circ} \mathrm{C}$ providing a level of confidence of approximately $95 \%$. This depicts of course the worst scenario, but it provides at least a first uncertainty on the temperature estimation. Since the expanded uncertainty of the IR measurement system was quite high, a systematic check with NTC thermistors in spot points was carried out during each experiment.

The effects of two significant uncertainty sources (dynamic response of each nail and gradient along the nail) can be easily included in the numerical simulation, thus allowing to reduce the expanded uncertainty to about $3^{\circ} \mathrm{C}$. The simulation presented in section 3 takes into account the heat transfer and the energy storage inside nails, balsa wood lid and active tip, other than the RF heating of the tissue. This also allows a direct comparison between IR surface measurements and simulated nails' temperatures.

The adopted RF generator (Invatec TAG 100W) works within the impedance range 20-300 $\Omega$ the procedure is automatically interrupted when the $Z$ exceeds the maximum value. The generator nominal power is usually different from the real power supplied to the sample: the latter was directly measured with the aid of an oscilloscope, a voltage probe and a current probe. Checks performed with saline through calorimetric measurements allowed verifying the reliability of the power values given by the oscilloscope: a systematic 5\% power reduction must be applied to obtain the real power supplied to the sample. During each test the power was set to $15 \mathrm{~W}$, corresponding to a real power supplied to the sample of about $14.2 \mathrm{~W}$ and held constant throughout the test.

\subsection{Experimental results}

The results for the configuration without insulating lid are used to determine the qualitative evolution of the thermal field close to the active tip. On the other hand the results from the setup with balsa wood lid provided data useful for the validation of mathematical model of the RFTA procedure and for evaluating the reliability of the experimental measurements with nail-equipped lid. During the first test the aforementioned heat losses greatly affect the procedure duration that is doubled (289 s) with respect to the same test with insulating lid (145 s). In order to check replicability, the test with nail-equipped lid was repeated a few times obtaining similar results.

The frames of Fig. 3 show some IR images from the "free surface" test, selected at proper times, to qualitatively outline the thermal field development. The frames can be grouped into two sets: a), b) and c) describe the temperature evolution before the heating effects can be visually observed, while d), e) and f) depict situations also visually detectable from the tissue color change produced by protein denaturation

Fig. 3(a) shows the situation at the heating onset $(t=10 \mathrm{~s})$ : two separate areas $\left(\sim 30^{\circ} \mathrm{C}\right)$ appear, where the active tip ends are positioned. The temperature increase is slightly higher at the right-hand end, where the stem tip is located. This asymmetry is observed throughout the test. Fig. 3(b) reproduces the observed situation at $t=42 \mathrm{~s}$ : the temperature distribution is getting more uniform along the active tip and the maxima at the two ends increase $\left(\sim 50^{\circ} \mathrm{C}\right)$. No tissue color change is yet noticeable. Fig. 3(c) gives the situation at $\mathrm{t}=64 \mathrm{~s}$ : the two temperature peaks $\left(\sim 60^{\circ} \mathrm{C}\right)$ expand to meet halfway, towards the middle of active electrode, with a temperature distribution assuming a ribbon shape. At this stage a slight tissue color change can be detected near the active tip ends through naked-eye observation. Fig. 3(d) shows the situation at $\mathrm{t}=201 \mathrm{~s}$ : the temperature peak reaches $70^{\circ} \mathrm{C}$ and its distribution has an external elliptical shape. The temperature maxima at the ends of the tip are still asymmetric. Fig. 3(e) gives the situation at $\mathrm{t}=265 \mathrm{~s}$ : vaporization has started near the active tip $\left(\sim 100^{\circ} \mathrm{C}\right)$. Fig. 3(f) gives the situation at $\mathrm{t}=289 \mathrm{~s}$, when the electrical power is automatically switched off: two wider areas with temperature higher than $100^{\circ} \mathrm{C}$ are detected.
They are centered on the ends of the active tip, where the vapor slightly inflates the tissue, as also noticed through naked-eye observation.
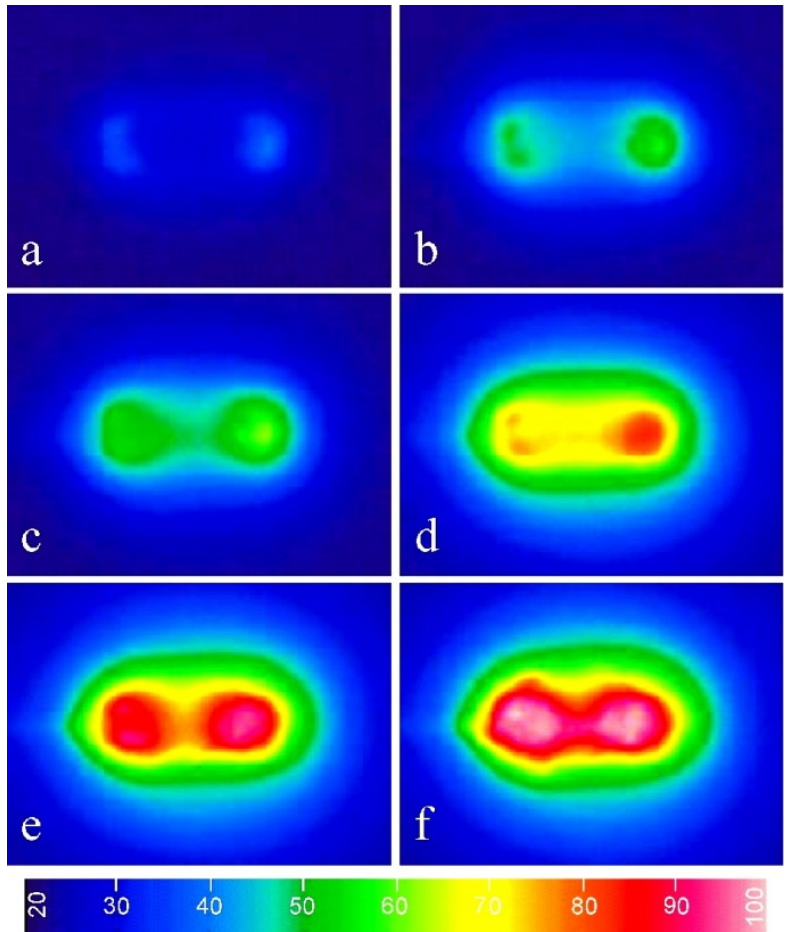

Fig. 3 Thermal field evolution ("free surface" test, IR images at different times): a) $\mathrm{t}=10 \mathrm{~s}$; b) $\mathrm{t}=42 \mathrm{~s}$; c) $\mathrm{t}=64 \mathrm{~s}$; d) $\mathrm{t}=201 \mathrm{~s}$; e) $t=265 \mathrm{~s}$; f) $t=289 \mathrm{~s}$ (just before power off). Color bar represents the temperature in degree Celsius.

The results of the insulated lid test are presented in the following. The temperature histories of nails regarded as the most meaningful are reported in Fig. 4, together with the results of the numerical simulation that will be discussed later.

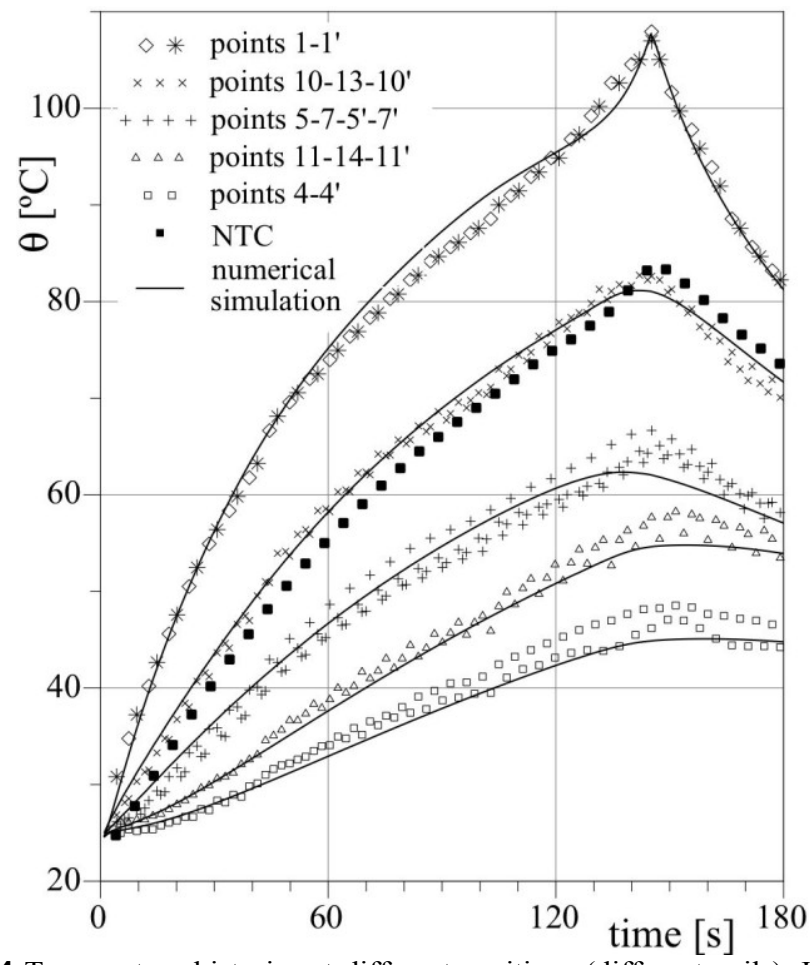

Fig. 4 Temperature histories at different positions (different nails). IR measurements (empty markers), NTC measurement (filled square markers) and numerical simulation results (lines). 
In this test the active electrode was fixed to the balsa wood lid and directly connected to nails $1-1$ ' as appears from Fig. 2. First of all a very good agreement of measurements from symmetrical points with respect to the nail pattern symmetry axis is observed (e.g., 1-1',10-10'). The overall symmetry is confirmed by comparing the NTC sensor measurements with the IR measurements of nails 10-10', whose position with respect to the symmetry axes of the active electrode is same (see Fig. 2). The symmetry of the thermal field distribution with respect to both symmetry axes of the active electrode highlights that effects of the heat losses due to the copper nails are negligible.

\section{NUMERICAL SIMULATIONS}

In this section, after briefly describing the mathematical model employed for the RFTA simulation, we will discuss the geometrical model used for the simulation of insulated lid test, the imposed boundary conditions and the model parameters, finally the simulations results will be presented and compared with experimental measurements.

\subsection{Mathematical model}

The temperature is computed solving the heat equation:

$$
\rho C_{p} \frac{\partial \theta}{\partial t}=\nabla \cdot(k \nabla \theta)+q
$$

where $\rho, C_{p}$ and $k$ are respectively density, specific heat at constant pressure and thermal conductivity. The source term $q$ is the heat produced by Joule effect per unit volume and is computed as:

$q=\frac{1}{\varepsilon_{r}}|\nabla V|^{2}$

where $\varepsilon_{r}$ is the electrical resistivity.

Adopting the quasi-static approximation, the electric field is computed by solving a quasi-harmonic equation for the electric potential $V$, obtained combining the continuity principle for the electric current and the local Ohm's law:

$\nabla \cdot\left(\frac{1}{\varepsilon_{r}} \nabla V\right)=0$

In the temperature range typical of RFTA $\left(37-120^{\circ} \mathrm{C}\right)$ the temperature dependence of liver tissue' physical parameters can be neglected (without losing accuracy) for the thermal parameters ( $\rho$,

$C_{p}$ and $k$ ) while it must be taken into account for the electrical parameters, sensibly affecting the simulation results (Rossi et al., 2008; Trujillo and Berjano, 2013). Therefore, both Eq. (1) and Eq. (3) must be solved iteratively at each time-step until convergence is achieved. The solution allows one to compute the impedance $\mathrm{Z}$, which is the variable used to stop the power supply as it occurs in the experimental situation. In order to obtain plausible results, the mathematical models used for the simulation of high-temperature hyperthermia treatments (e.g, RF, MW and laser ablation) must take into account the effects of vaporization. A review of the scientific literature suggests that Baldwin et al. (2001) were the first to investigate liquid-vapor phase change in thermal ablation treatments. The mathematical model proposed by Baldwin et al. (2001) was later reformulated and improved by Abraham and Sparrow (2007), using the enthalpy method to model the phase change, and applied to balloon thermal endometrial ablation, a method for the treatment of menorrhagia. Similar heuristic vaporization models were developed and applied to simulate high-temperature hyperthermia treatments (Yang et al., 2007; Ai et al., 2012; Gallati and Braschi,
2013). Since main purpose of the forthcoming numerical simulations is the validation of the measurement methodology, only the general features of the implemented phase change model will be discussed.

The liquid-vapor phase change of the tissue liquids is modeled using the Apparent Heat Capacity method (Bonacina et al., 1973; Muhieddine et al., 2011): the tissue specific heat is modified adding a symmetric Gaussian pulse to mimic the effects of the latent heat due to liquid vaporization. The pulse parameters $\mu$ and $\sigma$ (mean and standard deviation) control respectively the temperature corresponding to the Gaussian' peak and the temperature range over which the specific heat is modified.

During the phase change the tissue electrical resistivity is defined with the following empirical law:

$\varepsilon_{r}=\varepsilon_{r}\left(\theta_{v a p}\right)+\chi \psi(\theta)^{\tau}$

to model the effect of vapor bubbles that progressively insulate the active electrode leading to the procedure interruption. $\chi$ and $\tau$ are calibration parameters while $\psi(\theta)$ is the temperature integral of the Gaussian function (i.e., the relative amount of absorbed latent heat) that can be taken as the vapor volume fraction.

The thermal lesion boundary is approximated either using an Arrhenius damage model (Schutt and Haemmerich, 2008; Haemmerich and Schutt, 2011) or simply through a temperature isotherm; both $50^{\circ} \mathrm{C}$ (Mulier et al., 2012; Viglianti et al., 2014) and $60^{\circ} \mathrm{C}$ isotherms (Chang, 2010; Wood et al., 2011) are tested for the purpose. In the Arrhenius model the thermal damage $\Omega$ and the concentration of living cells $C$ are computed as follows (assuming $C(0)=1$ ):

$\Omega(t)=\int_{0}^{t} A e^{-\frac{E_{a}}{R^{*} \vartheta_{k}(t)}} d \xi$
$C(t)=\mathrm{e}^{-\Omega(t)}$

where $t$ is the time, $\theta_{k}$ is the absolute temperature $(\mathrm{K})$, $A=2.984 \cdot 10^{80}$ is the frequency factor $\left(\mathrm{s}^{-1}\right), E_{a}=5.064 \cdot 10^{5}$ is the activation energy $\left(\mathrm{Jmol}^{-1}\right)$ and $R^{*}$ is the universal gas constant $(8.314$ $\mathrm{Jmol}^{-1} \mathrm{~K}^{-1}$ ). A threshold value of 4.6 for $\Omega(t)$ (which corresponds to $99 \%$ cell death $-C(t)=0.01)$ is used to define the cell death boundary.

\subsection{Numerical model}

The equations of the mathematical model are discretized by means of the finite volume method using the $\mathrm{C}++$ library OpenFOAM ${ }^{\circledR}$. Equations (1) and (3) are solved with a fully implicit scheme to obtain the primary variables: $\theta$ and $V$. Second order accurate schemes are used for both time and space discretization.

Since one of main objectives was the validation of the experimental methodology we decided to build a complete threedimensional model of the testing box, simulating not only the RFTA on liver tissue but also the heat transfer inside lid, nails and active electrode. Exploiting the symmetry of the experimental setup only half of the testing box has been modeled, this helped reducing the computational cost without loss of generality. An exploded view of the three-dimensional geometrical model is depicted in Fig. 5 while some details are depicted in Fig. 6.

The computational domain is discretized into about $1.2 \mathrm{M}$ tetrahedral cells using the Delaunay-based volume grid generation method implemented in Gmsh (Geuzaine and Remacle, 2009). The mesh is manually refined where strong gradients of the primary variables are expected (i.e., close to the active electrode) and near the interfaces between different materials. The time-step is set to $1 \mathrm{~s}$, a 
sensitivity analysis of both mesh size and time-step has been carried out. The results later presented are obtained with a more refined mesh (about $2.8 \mathrm{M}$ cells) that gives smoother results close to the nail-lid interface. Equations (1) and (3) are solved in all the cells of the computational domain assigning appropriate electrical and thermal properties to the various materials.

The electric potential equation is solved assigning, as boundary conditions, a fixed electric potential to the passive electrode $(V=0)$ and to the cells belonging to the active electrode ( $V=V^{a e}$ ), while a noflux condition is imposed on the remaining boundaries. Since the impedance $\mathrm{Z}$ changes during the RFTA, an iterative procedure is needed to compute, at each time-step, the electric potential $V^{a e}$ required to supply a constant power to the tissue; for details see (Rossi et al., 2008). Furthermore a check is performed to control that all the electrical power is supplied to the liver tissue, avoiding erroneous thermal sources due to discretization and interpolation schemes.

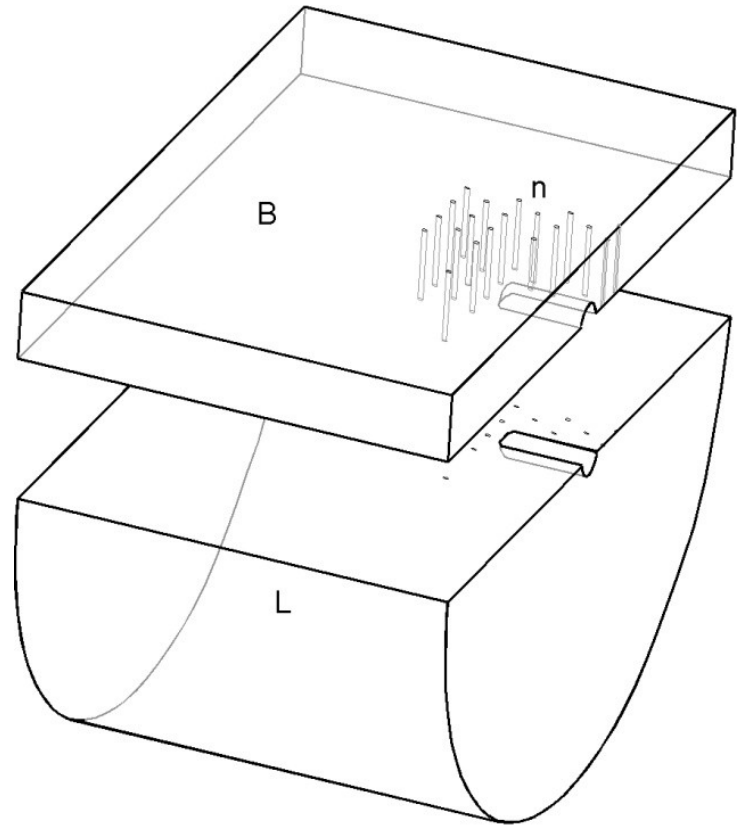

Fig. 5 3D geometrical model, exploded view. Liver tissue (L), balsa wood lid (B), nails (n).
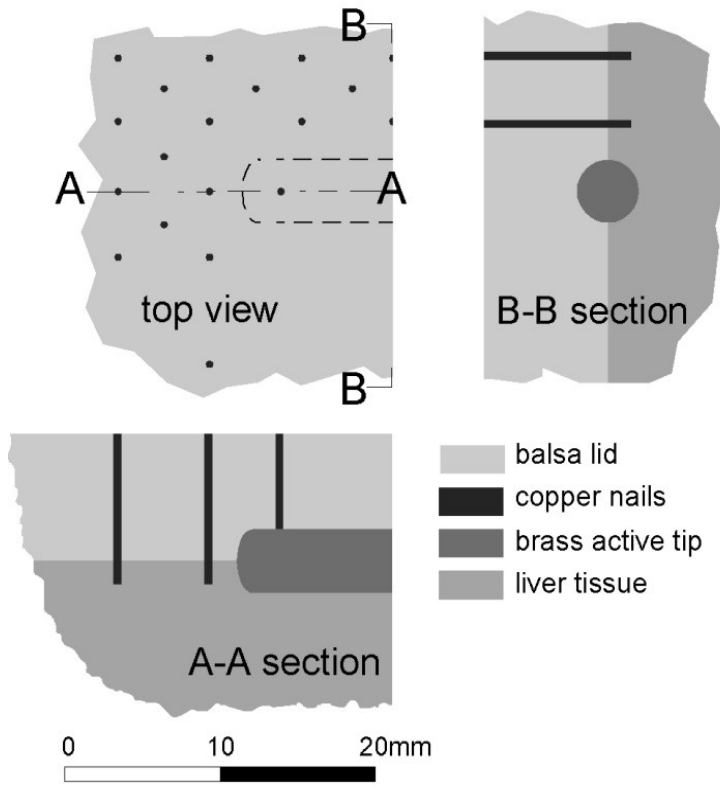

balsa lid copper nails brass active tip liver tissue

Fig. 6 Details of the computational model with material properties assignment.
Concerning the heat equation, no-flux boundary conditions are assigned to all boundaries except for the balsa lid and nails boundaries where the following convective boundary condition (accounting for the heat losses) is imposed:

$-k \nabla \theta \cdot \boldsymbol{n}=\alpha\left(\theta-\theta^{a m b}\right)$

where $\theta^{a m b}$ is the ambient temperature, $\alpha$ is a heat transfer coefficient and $\boldsymbol{n}$ is the outward unit normal vector.

In the simulation the initial conditions for Eq. (1) are set using the temperatures measured before the starting the RFTA experiment: $28^{\circ} \mathrm{C}$ for balsa wood lid and $23^{\circ} \mathrm{C}$ for liver tissue, nails and active electrode. Similarly the power set in the simulation is $14.2 \mathrm{~W}$ and the ambient temperature is $28^{\circ} \mathrm{C}$.

\subsection{Physical and model parameters}

Regarding the physical parameters standard literature values have been used for all the modeled materials (liver tissue, balsa wood lid, copper nails and brass active electrode). Most physical parameters are kept constant during the simulation; only the temperature dependence of the tissue electrical resistivity is taken into account since it greatly affects the tissue heating. The resistivity is computed from the temperature using an experimental curve determined for ex vivo porcine liver tissue (Rossi et al., 2008). It prescribes a significant variation $(55 \%$ in the temperature range $30-85^{\circ} \mathrm{C}$ ) following approximately a quadratic law (Zurbuchen et al., 2010). The experimental curve, normalized with the liver tissue resistivity at $40^{\circ} \mathrm{C}$, can be adequately interpolated by a $4^{\text {th }}$ degree polynomial:

$\varepsilon_{r} / \varepsilon_{r}^{40}=a_{0}+a_{1} x+a_{2} x^{2}+a_{3} x^{3}+a_{4} x^{4}$

where: $x=(\theta-40), a_{0}=2.5, a_{1}=-9.13 \cdot 10^{-2}, a_{2}=2.178 \cdot 10^{-3}$, $a_{3}=-2.495 \cdot 10^{-5}, a_{4}=1.029 \cdot 10^{-7}$.

The RFTA model has been previously calibrated using the ex vivo porcine liver tissue RF ablations performed by Gallati and Braschi (2013). The physical and calibration parameters are reported respectively in Table 1 and Table 2 . It is important to highlight that the values used for electrical resistivity of balsa wood, copper and brass are at least three orders of magnitude higher or lower than the liver tissue resistivity (Eq. 8 with $\varepsilon_{r}^{40}=3.3 \Omega \mathrm{m}$ ), their exact value does not affect the simulation results and therefore is not reported. The liquid-vapor phase change model employs the following physical parameters: water latent heat of vaporization $(2300 \mathrm{~kJ} / \mathrm{kg})$ and water content of liver tissue $(75 \%)$.

The heat transfer coefficient $\alpha$ is the only ad hoc parameter, nevertheless its value is low and therefore its effects are limited. It is introduced only in order to properly reproduce the heating and especially the cooling of the balsa wood lid. Because of their low value the losses do not sensibly affect the temperature distribution inside the tissue and the duration of the RFTA procedure.

During the phase change the resistivity must be significantly increased to model the electrical effect of vapor bubbles near the active electrode. The calibration parameters $\chi$ and $\tau$ control this increase; the sensitivity analysis proved that their variation affects even considerably the procedure duration and therefore also the temperature distribution. The calibration parameters $\sigma$ and $\mu$, that control the shape and position of the Gaussian function, affect both procedure duration (obviously also connected to the phase change) and the heating rate during vaporization; however only the latter has a significant effect. Several functions, other than the Gaussian adopted here, could have been used for the same purpose, with similar effects (provided that they subtend unit area). The sensitivity analysis proved that the main 
calibration parameters of the model are $\mu$ and $\tau$. These parameters are related to the phase change and their variation affects even strongly the procedure duration, that in turn affects the temperature gradient. However, the model analysis also highlighted that relatively small differences in the calibration parameters values (even up to 20\%) do not substantially affect the simulation outcome.

Table 1 Physical parameters: density, specific heat at constant density and thermal conductivity for each material considered in the simulation.

\begin{tabular}{l|c|c|c}
\hline & $\begin{array}{c}\rho \\
\left(\mathrm{kg} / \mathrm{m}^{3}\right)\end{array}$ & $\begin{array}{c}C_{p} \\
\left(\mathrm{~J} / \mathrm{kg} \cdot{ }^{\circ} \mathrm{C}\right)\end{array}$ & $\begin{array}{c}k \\
\left(\mathrm{~W} / \mathrm{m} \cdot{ }^{\circ} \mathrm{C}\right)\end{array}$ \\
\hline Liver tissue & 1070 & 3500 & 0.65 \\
\hline Balsa wood lid & 150 & 2900 & 0.09 \\
\hline Copper nails & 8950 & 390 & 395 \\
\hline Brass active tip & 8500 & 380 & 115 \\
\hline
\end{tabular}

Table 2 Calibration parameters; the first four parameters are connected with the phase change and were previously calibrated using RFTA tests.

\begin{tabular}{c|c|c|c|c}
\hline $\begin{array}{c}\mu \\
\left({ }^{\circ} \mathrm{C}\right)\end{array}$ & $\begin{array}{c}\sigma \\
\left({ }^{\circ} \mathrm{C}\right)\end{array}$ & $\begin{array}{c}\chi \\
(\Omega \cdot \mathrm{m})\end{array}$ & $\begin{array}{c}\tau \\
(-)\end{array}$ & $\begin{array}{c}\alpha \\
\left(\mathrm{W} / \mathrm{m}^{2} \cdot{ }^{\circ} \mathrm{C}\right)\end{array}$ \\
\hline 110 & 6 & 500 & 2 & 20 \\
\hline
\end{tabular}

\subsection{Simulation results}

The numerical simulation results are compared with the experimental measurements in Fig. 4 that depicts the temperature history of selected nails. A good agreement can be observed for all the nails, in particular for those close to the active electrode, whose maximum temperature is higher. The simulation results also highlight that the temperature on the nails' external face (the one measured by IR imaging) is very close to the temperature inside the liver tissue as shown in Figs. 7-8 (maximum difference about $2^{\circ} \mathrm{C}$ ). The model also reproduces quite well the temperature space-time evolution of balsa wood as can be seen in Fig. 9, where numerical simulation results and IR images are compared.

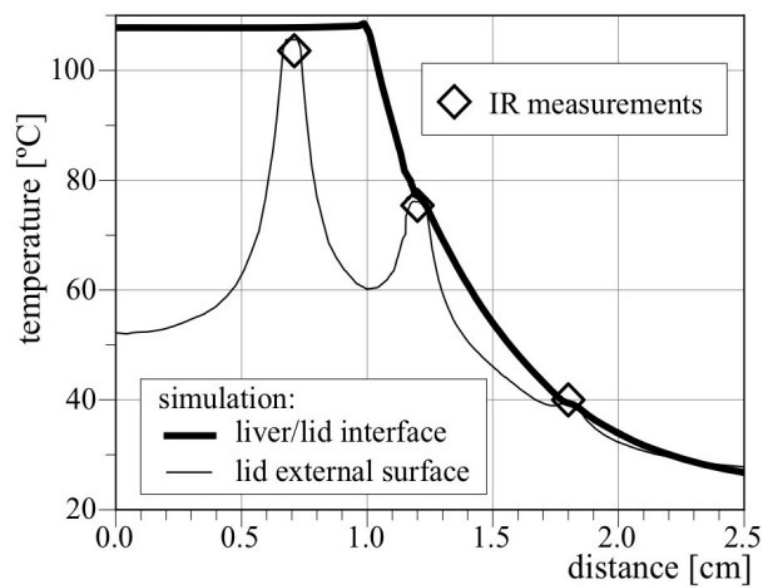

Fig. 7 Simulated axial temperature distribution $(\mathrm{t}=140 \mathrm{~s}$, along $\mathrm{x}$-axis of Fig. 2) in planes corresponding to the lid external surface (balsa wood lid with nails) and to the liver-lid interface. IR temperature measurements from nails 1', 6' and 17' are also plotted with empty markers.

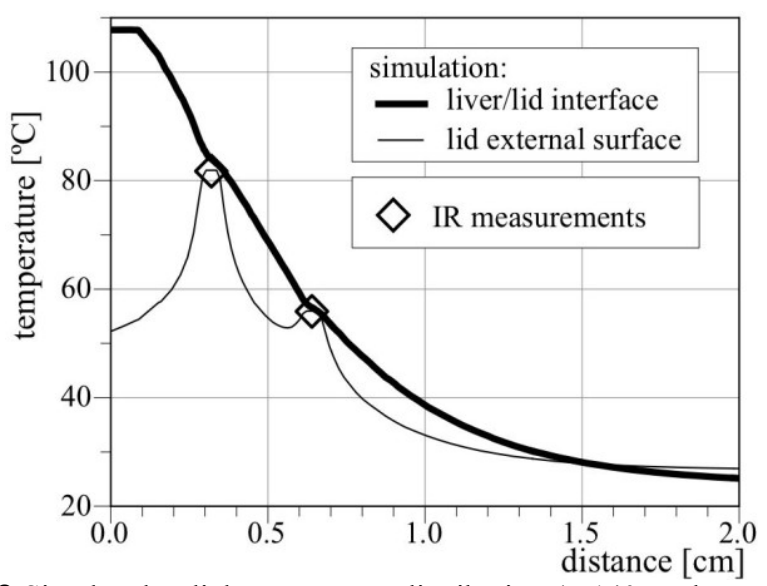

Fig. 8 Simulated radial temperature distribution $(\mathrm{t}=140 \mathrm{~s}$, along $\mathrm{y}$-axis of Fig. 2) in planes corresponding to the lid external surface (balsa wood lid with nails) and to the liver-lid interface. IR temperature measurements from the nails 13 and 14 are also plotted with empty markers.

IR images numerical simulation
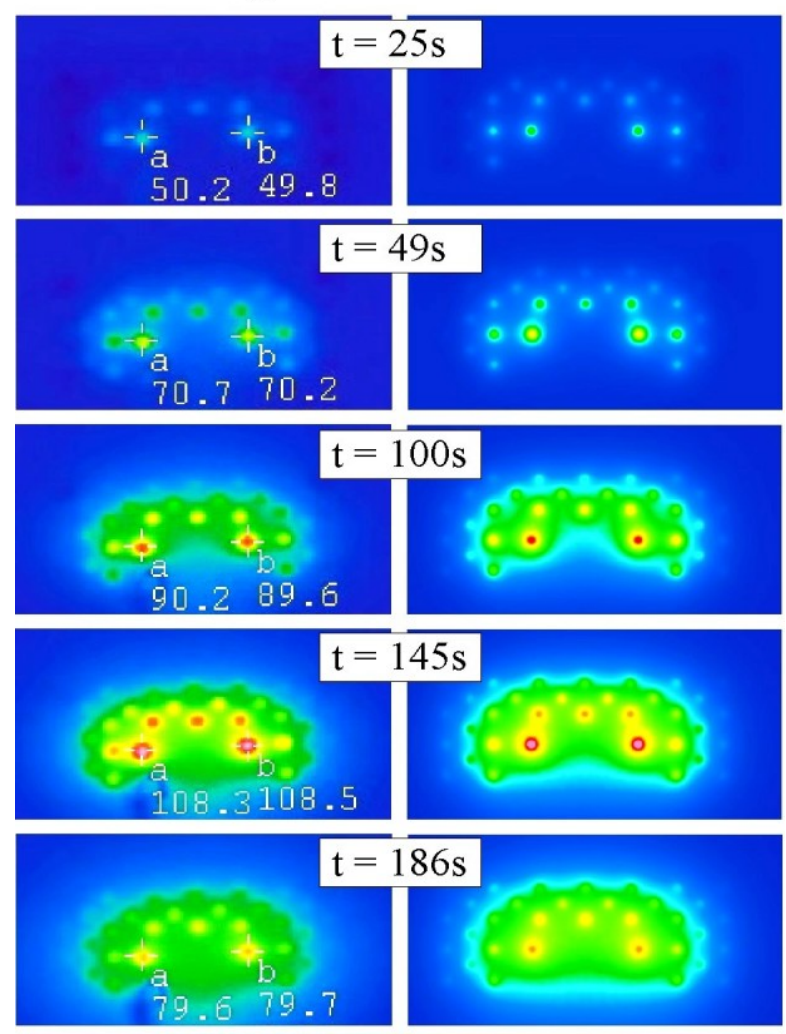

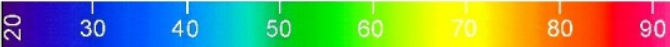

Fig. 9 Temperature space-time evolution (lid external surface): comparison between IR images (left-hand side) and numerical simulation results (right-hand side). The first four frames are during RFTA while the last frame $(\mathrm{t}=186 \mathrm{~s})$ is during cooling.

In the IR images the nails appear to be larger and hotter due to the nails' heads (diameter $1.3 \mathrm{~mm}$ ) that were not modeled because very thin. The difference may also be explained by the resolution of the IR measurements: the pixel dimensions are clearly higher than the cell size employed in the simulation for the nail discretization.

It is important to highlight that the asymmetry of the IR images (with respect to the active electrode longitudinal direction $-\mathrm{x}$-axis in Fig. 2) is due to the enhancement of the thermal losses on the side of the lid with the nails. This is also reproduced by the model as appears 
from Fig. 9 and Fig. 10(a) and clearly due to the copper nails presence. This asymmetry however does not sensibly alter the heating of liver tissue whose temperature remains symmetrical as can be seen from Fig. 10(b). On the other hand, the temperature distribution is always symmetric with respect to the transverse direction of the active electrode (y-axis in Fig. 2), as appears from Fig. 9. This is also confirmed by comparing the numerical simulation results of Fig. 10(b) with the ex vivo liver tissue thermal lesion (approximated by the tissue color change) obtained after performing the RFTA (Fig. 10(c)). The simulated thermal lesion boundaries, defined using Arrhenius model (Eqs. 5-6), $50^{\circ} \mathrm{C}$ isotherm and $60^{\circ} \mathrm{C}$ isotherm are compared with the experimental lesion, identified though tissue color change, in Fig. 10(c). In this experiment the simulated lesion, obtained using the Arrhenius model (dashed white line in Fig. 10), appears to be a better indicator for the thermal lesion.
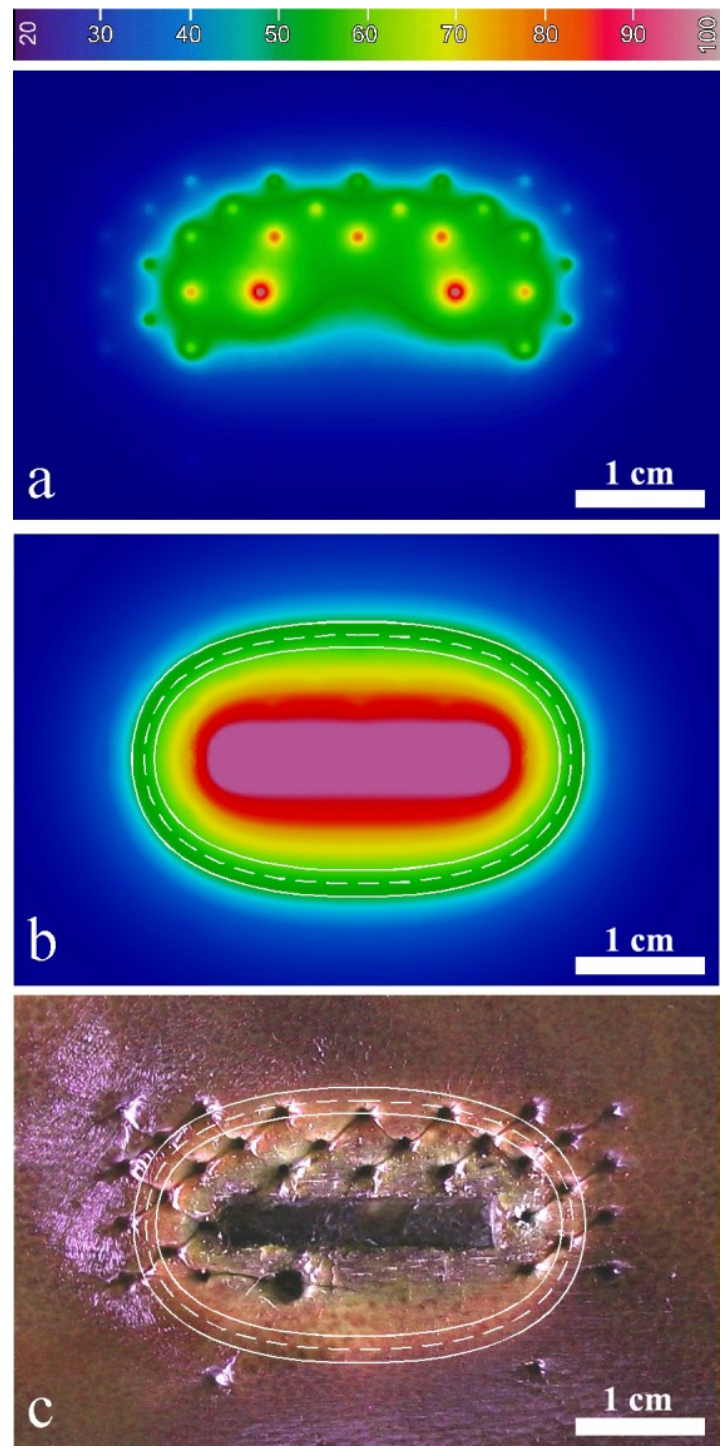

Fig. 10 Comparison between simulated temperature distribution (at the end of the procedure, $\mathrm{t}=140 \mathrm{~s}$ ) and experimental thermal lesion: a) lid external surface, simulation; b) liver tissue-lid interface, simulation; c) experimental thermal lesion due to protein denaturation. The white continuous lines in frames b) and c) represent the $50^{\circ} \mathrm{C}$ and $60^{\circ} \mathrm{C}$ isotherms obtained from the numerical simulation. The white dashed line identifies the thermal lesion obtained using the Arrhenius model.

In conclusion the numerical simulations results are in good agreement with the IR experimental measurements both from a qualitative and quantitative point of view. These results allowed the validation of the proposed measurement methodology that exploits an array of highly conductive nails and an insulating lid to measure the temperature in multiple points without altering significantly the phenomena. In addition these results confirm the soundness of the mathematical model, further contributing to its validation.

\section{CONCLUSIONS}

The space-time evolution of the thermal field was measured during RFTA on ex vivo porcine liver using thermal imaging. The experimental setup exploits an insulating lid equipped with an array of highly conductive small copper nails to allow multiple temperature measurements without altering the phenomena. The three-dimensional numerical simulation of the experiment, including the nail-equipped lid, allowed validating the temperature measurement methodology and further verifying the calibration of the proposed RFTA model. In future studies the proposed experimental methodology will be used for a thorough ex vivo experimental campaign using a similar setup. The most recent generation of distributed sensors, which make use of an interferometric setup to detect the Rayleigh backscattering within a single-mode optical fiber, are also under testing for temperature distribution measurements during RFTA. Further studies are underway for the development of a more physically-based model of liquid-vapor phase change exploiting a multiphase porous media theoretical framework.

\section{ACKNOWLEDGEMENTS}

The financial support of Ordine degli Ingegneri della Provincia di Pavia and Fondazione Cura Mini-invasiva Tumori ONLUS is gratetully acknowledged. The authors wish to thank Prof. Alfredo Cigada and $\mathrm{PhD}$ Lorenzo Comolli (Politecnico di Milano) for their assistance during IR measurements.

\section{NOMENCLATURE}

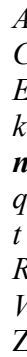

frequency factor $\left(\mathrm{s}^{-1}\right)$

specific heat at constant pressure $\left(\mathrm{Jkg}^{-1 \circ} \mathrm{C}^{-1}\right)$

activation energy $\left(\mathrm{Jmol}^{-1}\right)$

thermal conductivity $\left(\mathrm{Wm}^{-1}{ }^{\circ} \mathrm{C}^{-1}\right)$

outward unit normal vector $(-)$

Joule heat per unit volume $\left(\mathrm{Wm}^{-3}\right)$

time (s)

universal gas constant $\left(\mathrm{Jmol}^{-1} \mathrm{~K}^{-1}\right)$

electric potential $(\mathrm{V})$

electrical impedance $(\Omega)$

Greek Symbols

$\alpha \quad$ convective heat transfer coefficient $\left(\mathrm{Wm}^{-2 \circ} \mathrm{C}^{-1}\right)$

$\varepsilon_{r} \quad$ electrical resistivity $(\Omega \mathrm{m})$

$\theta \quad$ temperature $\left({ }^{\circ} \mathrm{C}\right)$

$\theta_{k} \quad$ absolute temperature $(\mathrm{K})$

$\mu \quad$ Gaussian function mean $\left({ }^{\circ} \mathrm{C}\right)$

$\rho \quad$ density $\left(\mathrm{kg} \mathrm{m}^{-3}\right)$

$\sigma \quad$ Gaussian function standard deviation $\left({ }^{\circ} \mathrm{C}\right)$

$\tau \quad$ parameter for phase change electrical resistivity (-)

$\chi \quad$ parameter for phase change electrical resistivity $(\Omega \mathrm{m})$

$\psi \quad$ vapor volume fraction (-)

Superscripts

amb ambient environment

ae active electrode

$40 \quad 40^{\circ} \mathrm{C}$ value

Subscripts

vap vaporization 


\section{REFERENCES}

Abraham, J.P. and Sparrow, E.M., 2007, “a Thermal Ablation Model Including Liquid-to-Vapor Phase Change, Necrosis-dependent Perfusion, and Moisture-dependent Properties," International Journal Heat and Mass Transfer, 50(13), 2537-2544.

http://dx.doi.org/10.1016/j.ijheatmasstransfer.2006.11.045

Ai, H., Wu, S., Gao, H., Zhao, L., Yang, C., Zeng, Y., 2012, "Temperature Distribution Analysis of Tissue Water Vaporization during Microwave Ablation: Experiments and Simulations," International Journal of Hyperthermia, 28(7), 674-685. http://dx.doi.org/10.3109/02656736.2012.710769

Baldwin, S.A., Pelman, A., Bert, J.L., 2001, "a Heat Transfer Model of Thermal Balloon Endometrial Ablation," Annals of Biomedical Engineering, 29(11), 1009-1018.

http://dx.doi.org/10.1114/1.1415521

Bonacina, C., Comini, G., Fasano, A., Primicerio, M., 1973, "Numerical Solution of Phase-change Problems," International Journal of Heat and Mass Transfer, 16(10), 1825-1832.

http://dx.doi.org/10.1016/0017-9310(73)90202-0

Chang I.A., 2010, "Considerations for Thermal Injury Analysis for RF Ablation Devices," The open biomedical engineering journal 4, 3-12. http://dx.doi.org/10.2174\%2F1874120701004020003

Gallati, M., and Braschi, G., 2013, "On the Simulation of Radio Frequency Thermal Lesions in Porcine Liver," Proceedings of the 32nd IASTED International Conference on Modelling, Identification and Control, Innsbruck, Austria.

http://dx.doi.org/10.2316/P.2013.794-032

Geuzaine, C., Remacle, J.-F., 2009, "Gmsh: a Three-dimensional Finite Element Mesh Generator with Built-in Pre- and Post-processing Facilities," International Journal for Numerical Methods in Engineering, 79(11), 1309-1331.

http://dx.doi.org/10.1002/nme.2579

Goldberg, S.N., Gazelle, G.S., Dawson, S.L., Rittman, W.J., Mueller, P.R., Rosenthal, D.I., 1995, "Tissue Ablation with Radiofrequency: Effect of Probe Size, Gauge, Duration, and Temperature on Lesion Volume," Academic Radiology, 2(5), 399-404.

http://dx.doi.org/10.1016/S1076-6332(05)80342-3

Haemmerich, D. and Schutt, D.J., 2011, "RF Ablation at Low Frequencies for Targeted Tumor Heating: In Vitro and Computational Modeling Results," IEEE Transactions on Biomedical Engineering 58(2), 404-410.

http://dx.doi.org/10.1109/TBME.2010.2085081

JCGM 100, 2008, Evaluation of Measurement Data - Guide to the Expression of Uncertainty in Measurement, BIPM, Sèvres, France.

http://www.bipm.org/en/publications/guides/gum.html

McGahan, J.P., Brock, J.M., Tesluk, H., Gu, W.Z., Schneider, P., Browning, P.D., 1992, "Hepatic Ablation with Use of Radiofrequency Electrocautery in the Animal Model," Journal of Vascular and Interventional Radiology, 3(2), 291-297.

http://dx.doi.org/10.1016/S1051-0443(92)72028-4

Muhieddine, M., Canot, É., March, R.J., Delannay, R., 2011, “Coupling Heat Conduction and Water-steam Flow in a Saturated Porous Medium," International Journal for Numerical Methods in Engineering, 85(11), 1390-1414.

http://dx.doi.org/10.1002/nme.3022
Mulier, S., Jiang, Y., Wang, C., Jamart, J., Marchal, G., Michel, L., Ni, Y., 2012, "Bipolar Radiofrequency Ablation with Four Electrodes: Ex vivo Liver Experiments and Finite Element Method Analysis. Influence of Inter-electrode Distance on Coagulation Size and Geometry," Internatonal Journal of Hyperthermia 28(7), 686-697.

http://dx.doi.org/10.3109/02656736.2012.706729

Rossi, S., Fornari, F., Pathies, C., Buscarini, L., 1990, "Thermal Lesions Induced by $480 \mathrm{kHz}$ Localized Current Field in Guinea Pig and Pig Liver," Tumori, 76(1), 54-57.

Rossi, S., Gallati, M., Braschi, G., Raboni, M., 2008, "Modelling the Liver RF Thermal Lesions", Proceedings of the 17th IASTED International Conference on Applied Simulation and Modelling, Corfu, Greece.

Schutt, D.J. and Haemmerich, D., 2008, "Effects of Variation in Perfusion Rates and of Perfusion Models in Computational Models of Radio Frequency Tumor Ablation," Medical Physics 35(8), 3462-3470. http://dx.doi.org/10.1118/1.2948388

Tosi, D., Macchi, E.G., Braschi, G., Gallati, M., Cigada, A., Poeggel, S., Leen, G., Lewis, E., 2014, "Monitoring of Radiofrequency Thermal Ablation in Liver Tissue Through Fibre Bragg Grating Sensors Array," Electronics Letters, 50(14), 981-983. http://dx.doi.org/10.1049/el.2014.0620

Trujillo, M., and Berjano, E., 2013, "Review of the Mathematical Functions Used to Model the Temperature Dependence of Electrical and Thermal Conductivities of Biological Tissue in Radiofrequency Ablation," International Journal of Hyperthermia, 29(6), 590-597. http://dx.doi.org/10.3109/02656736.2013.807438

Trujillo, M., Alba, J., Berjano, E., 2012, "Relationship Between Rolloff Occurrence and Spatial Distribution of Dehydrated Tissue during RF Ablation with Cooled Electrodes," International Journal of Hyperthermia, 28(1), 62-68.

http://dx.doi.org/10.3109/02656736.2011.631076

van Sonnenberg, E., McMullen, W., Solbiati, L., 2005, Tumor Ablation: Principles and Practice, Springer, New York, NY.

http://dx.doi.org/10.1007/0-387-28674-8

Viglianti, B.L., Dewhirst, M.W., Gorman, J.M., Abraham, J.P., Sparrow, E.M., 2014, "Rationalization of Thermal Injury Quantification Methods: Application to Skin Burns," Burns, 40(5), 896-902.

http://dx.doi.org/10.1016/j.burns.2013.12.005

Wood, M., Goldberg, S., Lau, M., Goel, A., Alexander, D., Han, F., Feinstein, S., 2011, "Direct Measurement of the Lethal Isotherm for Radiofrequency Ablation of Myocardial Tissue," Circulation: Arrhythmia and Electrophysiology 4(3), 373-378.

http://dx.doi.org/10.1161/CIRCEP.110.961169

Yang, D., Converse, M.C., Mahvi, D.M., Webster, J.G., 2007, "Expanding the Bioheat Equation to Include Tissue Internal Water Evaporation during Heating," IEEE Transactions on Biomedical Engineering, 54(8), 1382-1388.

http://dx.doi.org/10.1109/TBME.2007.890740

Zurbuchen, U., Holmer, C., Lehmann, K.S., Stein, T., Roggan, A., Seifarth, C., Buhr, H.J., Ritz, J.P., 2010, "Determination of the Temperature-dependent Electric Conductivity of Liver Tissue Ex Vivo and In Vivo: Importance for Therapy Planning for the Radiofrequency Ablation of Liver Tumours," International Journal of Hyperthermia, 26(1), 26-33. http://dx.doi.org/10.3109/02656730903436442 